\title{
Expression of PLAG1, HMGA1 and HMGA2 in minor salivary glands tumours
}

\author{
Ida Barca $^{1 \#}$, Chiara Mignogna ${ }^{2 \#}$, Giuseppe Donato ${ }^{2}$, Maria Giulia Cristofaro $^{1}$ \\ ${ }^{1}$ Department of Experimental and Clinical Medicine, Magna Græcia University, Catanzaro, Italy; ${ }^{2}$ Department of Health Science, Magna Græcia \\ University, Catanzaro, Italy \\ Contributions: (I) Conception and design: I Barca, C Mignogna; (II) Administrative support: None; (III) Provision of study materials or patients: MG \\ Cristofaro, G Donato; (IV) Collection and assembly of data: I Barca, MG Cristofaro; (V) Data analysis and interpretation: C Mignogna, G Donato; (VI) \\ Manuscript writing: All authors; (VII) Final approval of manuscript: All authors. \\ "These authors contributed equally to this work. \\ Correspondence to: Ida Barca. Department of Experimental and Clinical Medicine, University "Magna Græcia” of Catanzaro, Viale Europa - \\ Germaneto, 88100 Catanzaro, Italy. Email: barca.ida@gmail.com.
}

\begin{abstract}
Background: Diagnosis of minor salivary gland (MSG) tumours is often difficult, due to the scarce tissue obtained from bioptic excision and complex histopathological differential diagnosis. In our study we performed an immunohistochemical analysis of PLAG1, HMGA1 and HMGA2 on a series of MSG tumours, in order to develop a new helpful diagnostic panel.

Methods: A retrospective series of 17 surgical specimens of MSG tumours were analysed for the expression of PLAG1, HMGA1 and HMGA2. Three control cases were enrolled and analysed. An intensity and percentage-based approach was performed, creating a combined score panel.

Results: PLAG1 facilitate the diagnosis of benign tumours, discriminating it from malignant histotypes, with a defined cut-off value. Similarly, HMGA1 is significantly higher in benign histotypes than in malignant ones. HMGA2 in our series, did not reveal any association in identifying benign from malignant histotypes.

Conclusions: In this study we assessed the diagnostic role of PLAG1, HMGA1 and HMGA2 immunohistochemical analysis. The score panel facilitate histopathological diagnosis of these rare tumours, helping to distinguish benign tumours from malignant ones and ameliorating the differential diagnosis of specific histotypes.
\end{abstract}

Koywords: PLAG1; HMGA1; HMGA2; immunohistochemistry; minor salivary glands tumours (MSGs tumours)

Submitted Aug 15, 2020. Accepted for publication Jan 27, 2021.

doi: $10.21037 /$ gs-20-667

View this article at: http://dx.doi.org/10.21037/gs-20-667

\section{Introduction}

Minor salivary glands (MSG) tumours are rare neoplasia of the head and neck region. In 2016 the WHO classification of salivary glands tumours described 11 benign and 20 malignant histotypes (1). With rare exceptions, tumours involving major salivary glands may also arise in MSG through mucosal area of the upper aero-digestive tract, with the same histopathological features (2). Specific data about the incidence and clinical-pathological aspects of MSG tumours are usually difficult to collect, because most studies concerning salivary glands tumours include both major and MSGs $(3,4)$. TNM classification for MSG tumours are not included in major salivary glands classification, but instead at their anatomical site of origin (5). The incidence of each histotype is different, with a greater incidence of malignant histotypes in MSG (6). MSG tumours are

\footnotetext{
^ ORCID: 0000-0003-3182-5075.
} 
characterized by molecular heterogeneity that correspond to different clinical and histopathological properties. As known, a lot of genes are involved in the pathogenesis of cancers $(7,8)$. In MSG tumours, in particular, it's well known the role of pleomorphic adenoma gene 1 (PLAG1). PLAG-1 belongs to the zinc finger family of transcriptional factors, developmentally regulated, together with PLAG1like and PLAG2-like genes. It's a small gene located on chromosome 8q12 and it's implicated in specific types of tumours, particularly in pleomorphic adenoma (PLA) of the salivary glands which gives the name to the gene (9). Studies demonstrated that PLAG1expression is elevated during the embryonic/fetal period, whereas expression in most adult organs is low or absent, and this has led to the notion of PLAG1 being a 'fetal' transcription factor (10). A role for PLAG1 in tumour development was showed in different cancer such as lipoblastoma, hepatoblastoma, acute myeloid leukemia, and uterine leiomyoma and leiomyosarcoma (11). High mobility group A proteins family (HMGA proteins) are architectural transcriptional factors able to induce structural alterations of the chromatin. They were firstly isolated and characterized in 1973, when the electrophoresis of the chromatin of the thymus of a calf showed the presence of proteins able to migrate quickly inside the agarose gel (12). HMGA family is composed of four proteins: HMGA1a, HMGA1b, HMGA1c and HMGA2 (formerly HMGI, HMGY, HMG-I/R and HMGI-C, respectively). They are HMGA proteins can regulate the transcription of many genes in a positive or negative sense, directly modifying the DNA conformation binding Adenin-Timin rich regions, or indirectly interacting with other transcriptional factors(13). Many studies assign them important roles in regulation of proliferation, growth and differentiation of embryonal cells $(14,15)$. Due to their implication in many cellular mechanisms, an aberrant expression of HMGA proteins characterize a great number of human neoplasia, specially malignant $(16,17)$. The aim of our study is to obtain a new diagnostic and prognostic immunohistochemical panel finalized to the evaluation of PLAG1, HMGA1 and HMGA2. Histopathological diagnosis of MSG tumours is often difficult due to the scarce tissue sampling, frequently obtained from incisional biopsy and/or fine needle aspiration biopsy, with no data about tumour's peripheral area. Therefore the diagnostic process results difficult and immunohistochemical analysis are usually needed $(18,19)$. A new immunohistochemical panel will be useful in the clinical practice for diagnostic purposes, also suggesting new therapeutic targets. We present the following article in accordance with the MDAR reporting checklist (available at http://dx.doi.org/10.21037/gs-20-667).

\section{Methods}

\section{Patients}

Study population consisted of 17 patients with primary MSG tumours ( 7 men and 10 women; mean age: 56.4 years; range, 16-82 years). Surgical resection specimens were retrieved from the archive files of the Department of Health Sciences, Pathology Section, University Magna Graecia of Catanzaro, Italy. Bioptic samples of three patients with suspected Sjögren syndrome were included in the study, as normal controls. All patients undergone surgery at the Unit of Maxillo-Facial Surgery of University Magna Graecia of Catanzaro, from March 2010 to August 2014. The study protocol was approved by the Ethics Committee of Magna Graecia University of Catanzaro (reference number 146 of 20 May 2016) and was conducted in accordance with the "Ethical Principles for Medical Research Involving Human Subjects" described in the Helsinki Declaration (as revised in 2013); informed consent was obtained from the subjects for use of their tissue.

\section{Experimental procedures}

For all cases, histopathological diagnosis and tumour grading was confirmed jointly by two pathologists (GD, CM) by re-evaluation of the original Hematoxylin-Eosin (H\&E) stained sections of tumours. The histopathological series was constituted by the following histotypes: PLA $(n=6)$, mucoepidermoid carcinoma $(n=4)$, adenoid-cystic carcinoma $(n=4)$, polymorphic low grade adenocarcinoma $(n=2)$, carcinoma ex-pleomorphic adenoma $(\mathrm{n}=1)$.

For each case, $4 \mu \mathrm{m}$-thick serial sections were obtained from a representative block of formalin-fixed, paraffinembedded tissue, mounted on coated glass slides, and heated at $60^{\circ} \mathrm{C}$ for $60 \mathrm{~min}$.

A standard protocol for immunohistochemistry using an automated immunostainer (Bond ${ }^{\mathrm{TM}}$ Max; Leica Biosystems, Melbourne, Australia) was performed using the following primary antibodies: rabbit, polyclonal anti-PLAG1 (1:50 dilution, Abcam, Milano), anti-HMGA1 (1:200 dilution, Santa Cruz, Segrate, Milano) and anti-HMGA2 (1:200 dilution, Santa Cruz, Segrate, Milano). Negative control sections for immunohistochemistry were processed without 

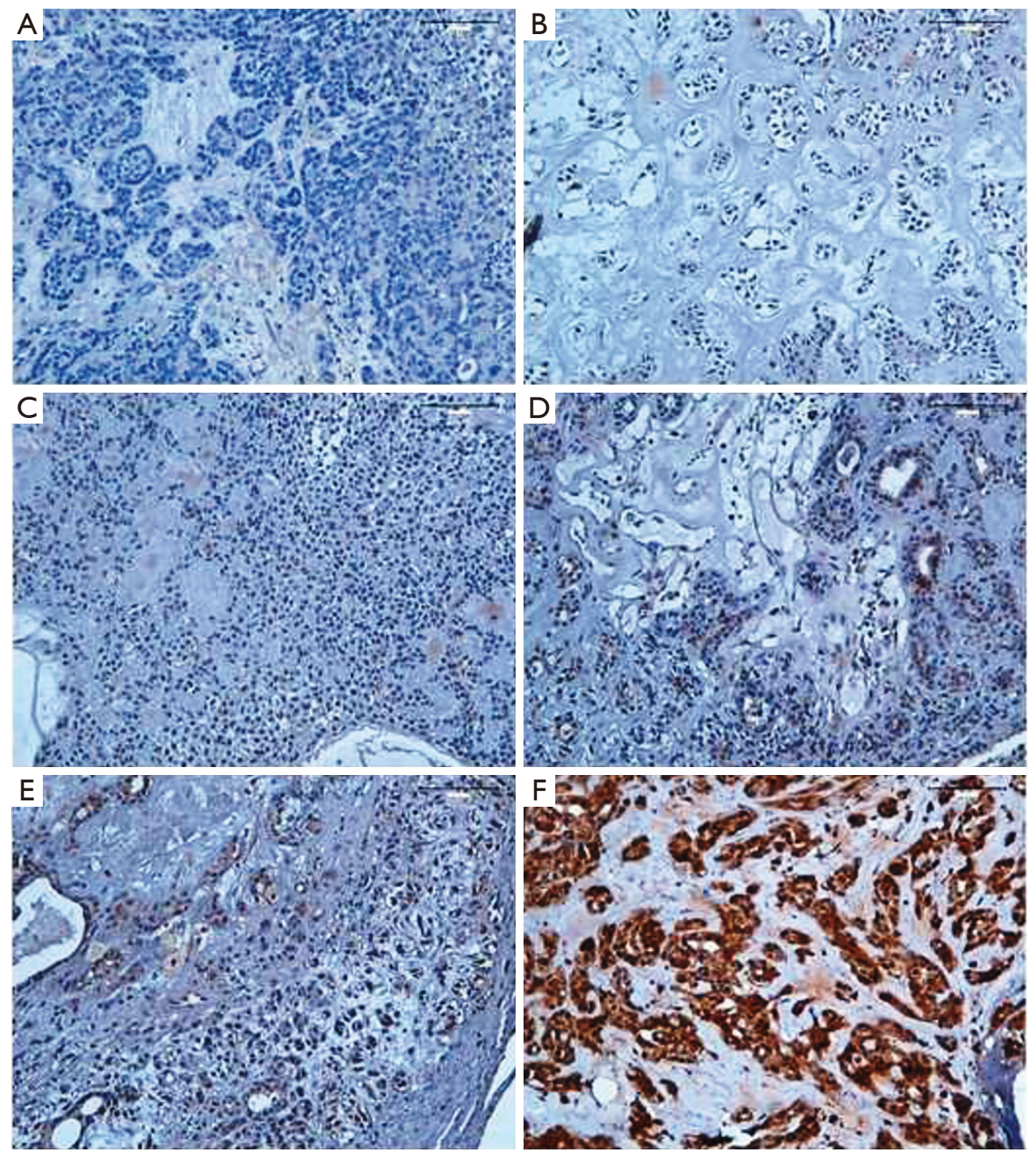

Figure 1 Percentage-based approach. Immunohistochemical analysis of PLAG1, HMGA1, HMGA2 in H\&E stained sections of minor salivary glands (MSG) tumours. The panel shows examples of proportion score assigned on the basis of the percentage of positive tumour cells. Score 0: no positive cells, in a case of Sjögren syndrome (A), Score 1: <1\% (B), Score 2: 1-10\% (C), Score 3: 10-33\% (D), Score 4: 33-66\% (E), Score 5: 66-100\% (F). Magnification 200x.

the primary antibody.

\section{Measures and outcomes}

A semi-quantitative analysis was performed, by the evaluation of both percentage of positive cells and staining intensity (intensity and percentage-based approaches), using the score system by Allred et al. modified. A percentage- based approach was used in order to estimate the proportion of positively stained tumour cells ( 0 : none, $1:<1 \%, 2$ : 1-10\%, 3: 10-33\% 4: 33-66\%, 5: 66-100\%) (Figure 1). Average estimated intensity of staining in positive cells was assigned as an intensity score $(0=$ no signal; $1=$ weak; $2=$ intermediate; $3=$ strong) (Figure 2). Proportion score and intensity score were added to obtain a total score (Sum Score) ranging from 0 to 8 . 

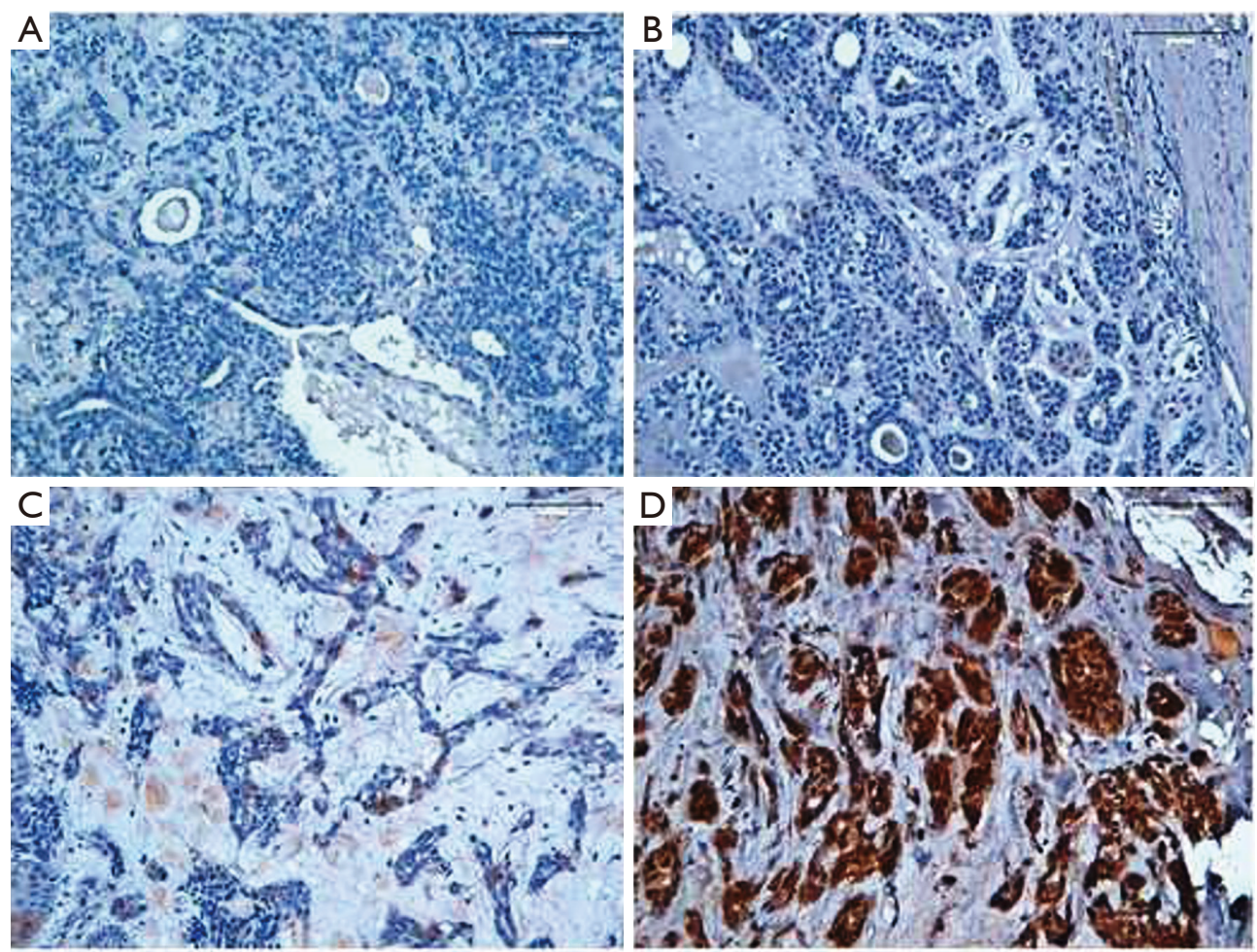

Figure 2 Intensity-based approach. Immunohistochemical analysis of PLAG1, HMGA1, HMGA2 in H\&E stained sections of minor salivary glands (MSG) tumours. The panel shows examples of different intensity score value: Score 0: signal absent (A); Score 1= weak (B); Score $2=$ intermediate (C); Score 3= strong (D). Magnification 200x.

\section{Statistical analysis}

Statistical Analysis was performed using non-parametric Mann-Whitney test (SPSS STATISTICS 21.0.0.1, IBM Corp, Armonk, NY, USA) because the sample was not homogeneous. Continuous variables were expressed by mean and standard deviation and were therefore compared. The level of significance was set to $\mathrm{P}<0.05$.

\section{Results}

The semi-quantitative analysis values of the processed specimens of both neoplastic and healthy glandular tissues (controls) are shown in Table 1. PLAG1 total score (intensity and percentage approach) was $5.3 \pm 0.5$ in benign tumours (PLA) and $3.6 \pm 0.5$ in malignant tumours $(\mathrm{P}<0.0001)$. Similarly, PLAG1 percentage score was respectively $4 \pm 0 v$ s. $2.5 \pm 0.5(\mathrm{P}<0.0001)$.

HMGA1 total score (intensity and percentage approach) was $4.8 \pm 0.4$ in benign tumours (PLA) and $4.5 \pm 0.7$ in malignant tumours $(\mathrm{P}=0.96)$. On the other hand, HMAG1 percentage score was respectively $3.6 \pm 0.5$ vs. $3.09 \pm 0.5$ $(\mathrm{P}<0.05)$.

HMGA2 total score (intensity and percentage approach) and percentage score were not significantly associated to histotype: respectively $6.0 \pm 1.6$ in benign tumours (PLA) and $5.09 \pm 1.3$ in malignant tumours $(\mathrm{P}=0.28)$; and $3.6 \pm 1.0$ in benign tumours (PLA) and $3.1 \pm 0.7$ in malignant tumours $(\mathrm{P}=0.182)$. The Sum Score obtained adding the results of PLAG1, HMGA1 and HMGA2 was $15 \pm 1.4$ in benign tumours (PLA) and 13.6 \pm 1.4 in malignant tumours $(\mathrm{P}=0.0003)$. None of the analysed markers was expressed in our series normal controls. Results of statistical analysis are showed in Table 2. No statistical correlation was highlighted between clinical stage and scores.

\section{Discussion}

Salivary gland tumours account for about $1-4 \%$ of all cancers and $5.3 \%$ of head and neck tumours $(1,3)$. Generally these neoplasms affect the major salivary glands and only in $12-30 \%$ of cases the MSG (20). However, 
Table 1 Immunohistochemical results

\begin{tabular}{|c|c|c|c|c|c|}
\hline Histotype & Stage & Score PLAG $_{1}$ & Score $\mathrm{HMGA}_{1}$ & Score $\mathrm{HMGA}_{2}$ & Sum Score \\
\hline Pleomorphic adenoma & NA & $(4+1)=5$ & $(4+1)=5$ & $(4+3)=7$ & 17 \\
\hline Pleomorphic adenoma & NA & $(4+2)=6$ & $(4+1)=5$ & $(3+1)=4$ & 15 \\
\hline Pleomorphic adenoma & NA & $(4+1)=5$ & $(3+1)=4$ & $(4+3)=7$ & 16 \\
\hline Pleomorphic adenoma & NA & $(4+1)=5$ & $(4+1)=5$ & $(2+2)=4$ & 16 \\
\hline Mucoepidermoid carcinoma & T1 No Mo I & $(3+1)=4$ & $(3+2)=5$ & $(3+1)=4$ & 13 \\
\hline Mucoepidermoid carcinoma & T2 NO Mo II & $(3+1)=4$ & $(2+2)=4$ & $(3+2)=5$ & 13 \\
\hline Mucoepidermoid carcinoma & T2 NO MO II & $(3+1)=4$ & $(3+1)=4$ & $(3+2)=5$ & 13 \\
\hline Adenoid cystic carcinoma & T2 N0 MO II & $(3+1)=4$ & $(3+2)=5$ & $(2+2)=4$ & 13 \\
\hline Adenoid cystic carcinoma & T3 NO MO III & $(2+1)=3$ & $(4+2)=6$ & $(3+2)=5$ & 14 \\
\hline Adenoid cystic carcinoma & T1 No Mo I & $(2+2)=4$ & $(3+2)=5$ & $(3+2)=5$ & 14 \\
\hline Polymorphic low grade adenocarcinoma & T2 No MO II & $(3+1)=4$ & $(3+2)=5$ & $(5+3)=8$ & 17 \\
\hline Polymorphic low grade adenocarcinoma & T3 N1 M0 III & $(2+1)=3$ & $(3+1)=4$ & $(4+3)=7$ & 14 \\
\hline Carcinoma ex-pleomorphic adenoma & T3 N1 M0 III & $(3+1)=4$ & $(3+1)=4$ & $(3+1)=4$ & 12 \\
\hline Control 1 & NA & $(0+0)=0$ & $(0+0)=0$ & $(0+0)=0$ & 0 \\
\hline Control 2 & NA & $(0+0)=0$ & $(0+0)=0$ & $(0+0)=0$ & 0 \\
\hline
\end{tabular}

$\mathrm{PLAG}_{1}$, pleomorphic adenoma gene 1; $\mathrm{HMGA}_{1}$, high mobility group AT-hook 1; HMGA , high mobility group AT-hook 2.

Table 2 Immunohistochemical analysis

\begin{tabular}{lccc}
\hline Immunohistochemical analysis & Benign & Malignant & P \\
\hline PLAG1 percentage score & $4 \pm 0$ & $2.5 \pm 0.5$ & $<.5001$ \\
HMGA1 percentage score & $3.6 \pm 0.5$ & $3.09 \pm 0.5$ & 0.05 \\
HMGA2 percentage score & $3.6 \pm 1.0$ & $3.1 \pm 0.7$ & $<.182$ \\
PLAG1total score & $5.3 \pm 0.5$ & $4.5 \pm 0.7$ & 0.0001 \\
HMGA1 total score & $4.8 \pm 0.4$ & $5.09 \pm 1.3$ & 0.28 \\
HMGA2 total score & $6.0 \pm 1.6$ & $13.6 \pm 1.4$ & 0.0003 \\
Sum Score & $15 \pm 1.4$ & \\
\hline
\end{tabular}

recent studies showed a most common development of malignant tumours in MSG respect to major salivary gland $(2,3)$. Emerging data from the literature demonstrated a close relationship between prognosis and stage of MSG cancers (6). It is noteworthy that surgical resection of MSG, practiced as excisional or incisional biopsy, often provides scarce tissue available for histological analysis, therefore immunohistochemical expression of control 
samples. Our aims were: check the diagnostic value of immunohistochemical expression of single markers and their combination (Sum Score) in a group of rare and difficult to assess tumours; provide new information on the etiopathogenesis of these uncommon tumours. PLAG1 immunohistochemical expression in salivary gland tumours is controversial $(21,22)$. Previous translational studies on cell cultures from benign and malignant salivary gland tumours suggest that the expression of PLAG1 can be considered an hallmark of these neoplasms; accordingly, the PLAG1 mRNA was reported in all histotypes of the salivary glands tumours. Our results showed that the semiquantitative evaluation of PLAG1 (intensity and percentage score) can be useful in the differential diagnosis between benign and malignant tumours. In particular, we have determined a cutoff value ( $>4)$ that is able to identify benign histotypes (PLA) from malignant ones. To be noted, PLAG1 percentage score $>4$ is anyway statistically associated to benign tumours (PLA). We described for the first time, the expression pattern of HMGA1 in both benign and malignant MSG tumours. HMGA1 overexpression has been reported in many cancer types, including pancreas, thyroid, colon, breast, lung, ovary, uterine cervix and body, head and neck tumours. Moreover, HMGA1 expression increased gradually from benign to malignant histotype, as in the case of astrocytoma evolution to glioblastoma, showing a strong correlation with the histologic grade (17). In our series, the percentage of cells expressing HMGA1 appears higher in benign histotypes (PLA) than in malignant ones $(\mathrm{P}<0.05)$. In this case, we can speculate that HMGA1 exerts a role in cell differentiation, acting as tumour suppressor. Accordingly, a dual effect of HMGA1 on cell transformation has been previously described, for which HMGA1 transforming properties seem to be context- and cell type-dependent (23).

HMGA2 alteration has been reported in both benign and malignant tumours. Literature data highlighted a positive correlation between HMGA2 expression and invasive and metastatic capacity in colon, breast and lung carcinoma (24). Interestingly, a causal role for HMGA2 in carcinogenesis and tumour progression has been showed in many cancer types, including colon and rectum, breast, pancreatic, ovarian, lung, testicular and oral cancer $(25,26)$. In our series, no useful information can be obtained from HMGA2 expression for differential diagnosis between benign and malignant histotypes. However, HMGA2 total score seems to be useful for differential diagnosis between Polymorphic Low-grade Adenocarcinoma (score 7-8) and AdenoidCystic Adenocarcinomas (score 4-5). The first histotype is typically described in oral cavity; it is one of the most difficult cancers to be diagnosed, due to the polymorphic microscopic appearance, morphologically resembling the more aggressive Adenoid-Cystic Carcinomas. So this marker can be useful in doubtful cases. Overall, our results suggest that the Sum Score of the three markers can be useful to distinguish benign tumours (PLA) from malignant histotypes, providing the pathologist with a new useful diagnostic tool. This immunohistochemical panel will be precious above all in cases where tissue samples are scarce as in MSG bioptic excision. To be noted, oncogenic capacity of PLAG1 has been largely described both in vitro and in vivo. Molecular mechanisms involved in this oncogenic activity include, at least in part, cellular signalling triggered by anti-apoptosis insulin-like growth factor 2 (IGF-2) and IGF-1R (27). IGF-1R is one of the upstream receptor tyrosine kinase responsible for activating the two major cascades, PI3K/AKT/mTOR and MAPK reported in human cancer (28). Moreover, IGF-1R is associated to tumorigenesis, metastasis, and therapy resistance (29). A very high expression of IGF-2 has been reported in PLAs over-expressing PLAG1. In particular, PLAG1 promotes tumorigenesis through the mitogenic action of IGF-2, mediated by the Ras/Raf/MAPK signalling pathway, or by binding Bcl-2 which blocks its function in the activation of PI3k/Akt proapoptotic pathway (30). As far as HMGA proteins is concerned, oncogenic activity is mediated by several mechanisms: induction of E2F1 and AP1 activity, induction of cyclin A expression, inactivation of p53induced apoptosis, impairment of DNA repair, enhancement of expression of proteins involved in inflammation and modulation of the expression of microRNAs and genes involved in epithelial-mesenchymal transition (31). Interestingly, the biological activity of HMGA proteins can be also modulated in response to hormonal and nutritional changes, recognized as IGF-1R-associated stressful conditions (32). Chiefari et al. [2012] indicate HMGA1 as a downstream target of the insulin receptor signalling pathway. HMGA1 in turn regulates IGFBP-1 and IGF$1 \mathrm{R}$ expression, via both the inhibition of $\mathrm{p} 53$ and the enhancement of Sp1 stimulatory activity. Previous studies showed that HMGA2 could regulate the transcription of IGF2BP2 during embryonic development as well as in controlling myoblast proliferation (33). Additionally, two independent study suggest that the HMGA2-IGF2BP2 pathway plays a crucial role to maintain proliferation and survival, perhaps by blocking differentiation mechanisms, in particular settings of cancer (34). Altogether, literature 
data indicate that oncogenic activity of PLAG1, HMGA1 and HMGA2 require the activation of insulin receptor signalling, however the precise molecular mechanisms are not fully elucidated. Even though the expression of these markers is always associated to neoplastic transformation (as confirmed by the negativity reported in MSG control tissues), the variable expression in benign and malignant tumours strongly suggest the involvement of IGF1Rindipendent pathways.

\section{Conclusions}

In conclusion, our study we performed an immunohistochemical analysis of PLAG1, HMGA1 and HMGA2 on a series of MSG tumours, in order to develop a new helpful diagnostic panel. This work is continuing with new cases to validate the protocol also because the investigation of signaling pathways that are activated by PLAG1, HMGA1 and HMAGA2, could lead to the identification of new therapeutic targets. Additionally, molecules and nanoparticles displaying anti-HMGA1 (nitrospina, distamycin, berenil) (35) and anti-HMGA2 (doxorubicin, siRNA, the analog of curcumin EF24) activities, already described in literature $(36,37)$, could be used in the future in addition to traditional therapies.

\section{Acknowledgments}

Funding: None.

\section{Footnote}

Reporting Checklist: The authors have completed the MDAR reporting checklist. Available at http://dx.doi.org/10.21037/ gs-20-667

Data Sharing Statement: Available at http://dx.doi. org/10.21037/gs-20-667

Conflicts of Interest: All authors have completed the ICMJE uniform disclosure form (available at http://dx.doi. org/10.21037/gs-20-667). The authors have no conflicts of interest to declare.

Ethical Statement: The authors are accountable for all aspects of the work in ensuring that questions related to the accuracy or integrity of any part of the work are appropriately investigated and resolved. The study protocol was approved by the Ethics Committee of Magna Graecia University of Catanzaro (reference number 146 of 20 May 2016) and was conducted in accordance with the "Ethical Principles for Medical Research Involving Human Subjects" described in the Helsinki Declaration (as revised in 2013); informed consent was obtained from the subjects for use of their tissue.

Open Access Statement: This is an Open Access article distributed in accordance with the Creative Commons Attribution-NonCommercial-NoDerivs 4.0 International License (CC BY-NC-ND 4.0), which permits the noncommercial replication and distribution of the article with the strict proviso that no changes or edits are made and the original work is properly cited (including links to both the formal publication through the relevant DOI and the license). See: https://creativecommons.org/licenses/by-nc$\mathrm{nd} / 4.0 \%$.

\section{References}

1. El-Naggar AK, Chan JKC, Grandis JR, et al. Tumours of salivary glands. In WHO Classification of Head and Neck Tumours, 4th Edition, IARC (International Agency for Research on Cancer) (ed) Lyon 2017:160-2.

2. Wyszyńska-Pawelec G, Gontarz M, Zapala J, et al. Minor salivary gland tumours of upper aerodigestive tract: a clinicopathological study. Gastroenterol Res Pract 2012;2012:780453.

3. Fonseca FP, Carvalho Mde V, de Almeida OP, et al. Clinicopathologic analysis of 493 cases of salivary gland tumors in a Southern Brazilian population. Oral Surg Oral Med Oral Pathol Oral Radiol 2012;114:230-9.

4. Barca I, Cristofaro MG. Surgical approach to parotid pleomorphic adenoma: a 15 -year retrospective cohort study. Br J Oral Maxillofac Surg 2020;58:659-62.

5. UICC (International Union Against Cancer). TNM classification of malignant tumours. 7th ed. Leslie $\mathrm{H}$. Sobin, Mary K. Gospodarowicz, Christian Wittekind (ed). New York, Chichester, Weinheim, Brisbane, Singapore, Toronto: Wiley - Blackwell, 2010.

6. Vaidya AD, Pantyaidya GH, Metgudmath R, et al. Minor salivary gland tumors of the oral cavity: a case series with review of literature. J Cancer Res Ther 2012;8:S111-115.

7. La Torre D, Maugeri R, Angileri FF, et al. Human leukocyte antigen frequency in human high-grade gliomas: A case-control study in Sicily. Neurosurgery 2009;64:1082-8. 
8. La Torre D, Conti A, Hammed Aguennouz M, et al. Telomere Length Modulation in Human Astroglial Brain Tumors. PLoS One 2013;8:e64296.

9. Rotellini M, Palomba A, Baroni G, et al. Diagnostic utility of PLAG1 immunohistochemical determination in salivary gland tumors. Appl Immunohistochem Mol Morphol 2014;22:390-4.

10. Juma AR, Damdimopoulou PE, Grommen SVH, et al. Emerging role of PLAG1 as a regulator of growth and reproduction. J Endocrinol 2016;228:R45-56.

11. Voz ML, Mathys J, Hensen K, et al. Microarray screening for target genes of the proto-oncogene PLAG1. Oncogene 2004;23:179-91.

12. Reeves R. Molecular biology of HMGA proteins: hubs of nuclear function. Gene 2001;277:63-81.

13. Cleynen I, Van de Ven WJ. The HMGA proteins: a myriad of functions (Review). Int $\mathrm{J}$ Oncol 2008;32:289-305.

14. Sumter TF, Xian L, Huso T, et al. The High Mobility Group A1 (HMGA1) Transcriptome in Cancer and Development. Curr Mol Med 2016;16:353-93.

15. Chiappetta G, Avantaggiato V, Visconti R, et al. High level expression of the HMGI $(\mathrm{Y})$ gene during embryonic development. Oncogene 1996;13:2439-46.

16. Melillo RM, Pierantoni GM, Scala S, et al. Critical role of the HMGI $(\mathrm{Y})$ proteins in adipocytic cell growth and differentiation. Mol Cell Biol 2001;21:2485-95.

17. Donato G, Martinez Hoyos J, Amorosi A, et al. High mobility group A1 expression correlates with the histological grade of human glial tumors. Oncol Rep 2004;11:1209-13.

18. Gonçalves AJ, Menezes MB, Kavabata NK, et al. Fine needle aspiration in salivary gland tumors: specifity and sensititivity. Rev Assoc Med Bras 2007;53:267-71.

19. Rimm DL, Stastny JF, Rimm EB, et al. Comparison of costs of Fine- Needle Aspiration and Open Surgical Biopsy as Methods for Obtaining a Pathologic Diagnosis. Cancer 1997;81:51-6.

20. Jaafari-Ashkavandi Z, Ashraf MJ, Afandak N. A clinicopathologic study of 82 intraoral minor salivary gland tumors. Iran Red Crescent Med J 2011;13:674-7.

21. Martins C, Fonseca I, Roque L, et al. PLAG1 gene alterations in salivary gland pleomorphic adenoma and carcinoma ex-pleomorphic adenoma: a combined study using chromosome banding, in situ hybridization and immunocytochemistry. Mod Pathol 2005;18:1048-55.

22. Matsuyama A, Hisaoka M, Nagao Y, et al. Aberrant
PLAG1 expression in pleomorphic adenomas of the salivary gland: a molecular genetic and immunohistochemical study. Virchows Arch 2011;458:583-92.

23. Li Y, Lu J, Prochownik EV. Dual role for SUMO E2 conjugase Ubc9 in modulating the transforming and growth-promoting properties of the HMGA1b architectural transcription factor. J Biol Chem 2007;282:13363-71.

24. Morishita A, Zaidi MR, Mitoro A, et al. HMGA2 is a driver of tumor metastasis. Cancer Res 2013;73:4289-99.

25. Miyazawa J, Mitoro A, Kawashiri S, et al. Expression of mesenchyme-specific gene HMGA2 in squamous cell carcinomas of the oral cavity. Cancer Res 2004;64:2024-9.

26. Pallante P, Sepe R, Puca F, et al. High mobility group A Proteins as Tumor Markers. Front Med (Lausanne) 2015;2:15.

27. Van Dyck F, Declercq J, Braem CV, et al. PLAG1, the prototype of the PLAG gene family: versatility in tumour development (review). Int J Oncol 2007;30:765-74.

28. LeRoith D, Roberts CT. The insulin-like growth factor system and cancer. Cancer Lett 2003;195:127-37.

29. Chitnis MM, Yuen JS, Protheroe AS, et al. The type 1 insulin-like growth factor receptor pathway. Clin Cancer Res 2008;14:6364-70.

30. van Maldegem AM, Bovée JV, Peterse EF, et al. Ewing sarcoma: The clinical relevance of the insulin-like growth factor 1 and the poly-ADP-ribose-polymerase pathway. Eur J Cancer 2016;53:171-80.

31. Fusco A, Fedele M. Roles of HMGA proteins in cancer. Nat Rev Cancer 2007;7:899-910.

32. Chiefari E, Nevolo MT, Arcidiacono B, et al. HMGA1 is a novel downstream nuclear target of the insulin receptor signaling pathway. Sci Rep 2012;2:251.

33. Cleynen I, Brants JR, Peeters K, et al. HMGA2 regulates transcription of the Imp2 gene via an intronic regulatory element in cooperation with nuclear factor-kappaB. Mol Cancer Res 2007;5:363-72.

34. Li Z, Zhang Y, Ramanujan K, et al. Oncogenic NRAS, required for pathogenesis of embryonic rhabdomyosarcoma, relies upon the HMGA2-IGF2BP2 pathway. Cancer Res 2013;73:3041-50.

35. Wegner M, Grummt F. Netropsin, distamycin and berenil interact differentially with a high- affinity binding site for the high mobility group protein HMG-I. Biochem Biophys Res Commun 1990;166:1110-7. 
36. Eivazy P, Atyabi F, Jadidi-Niaragh F, et al. The impact of the codelivery of drug-siRNA by trimethyl chitosan nanoparticles on the efficacy of chemotherapy for metastatic breast cancer cell line (MDA-MB-231). Artif Cells Nanomed Biotechnol 2017;45:889-96.

Cite this article as: Barca I, Mignogna C, Donato G, Cristofaro MG. Expression of PLAG1, HMGA1 and HMGA2 in minor salivary glands tumours. Gland Surg 2021;10(5):16091617. doi: $10.21037 /$ gs-20-667
37. Wu Z, Eguchi-Ishimae M, Yagi C, et al. HMGA2 as a potential molecular target in KMT2A-AFF1-positive infant acute lymphoblastic leukaemia. Br J Haematol 2015;171:818-29. 